\title{
Variations in maternal behavior in C57BL/6J mice: behavioral comparisons between adult offspring of high and low pup-licking mothers
}

\author{
Cort A. Pedersen ${ }^{1}$, Sivaramprasad Vadlamudi' , Maria L. Boccia ${ }^{2}$ and Sheryl S. Moy ${ }^{1,3}$ \\ 1 Department of Psychiatry, The University of North Carolina at Chapel Hill, Chapel Hill, NC, USA \\ 2 Frank Porter Graham Child Development Institute, The University of North Carolina at Chapel Hill, Chapel Hill, NC, USA \\ ${ }^{3}$ Carolina Institute for Developmental Disabilities, The University of North Carolina at Chapel Hill, Chapel Hill, NC, USA
}

Edited by:

Josephine Johns, University of North

Carolina at Chapel Hill, USA

\section{Reviewed by:}

Frances A. Champagne, Columbia University, USA

Sue Carter, University of Illinois at Chicago, USA

\section{*Correspondence:}

Cort A. Pedersen, Department of Psychiatry, The University of North Carolina at Chapel Hill, CB \#7160, Chapel Hill, NC 27599-7160, USA. e-mail:cort_pedersen@med.unc.edu
The amount of maternal licking received by newborn rats affects their adult stress reactivity and maternal behavior. Mouse studies in which litters were cross-fostered between strains that exhibit high vs. low amounts of maternal behavior also suggest that rearing conditions affect adult outcomes. The current study is the first to compare within a single mouse strain (C57BL/6J) behavioral responses between adult animals reared by mothers that exhibited frequencies of pup-licking (PL) at the high end and the low end of the normal distribution within the strain. Maternal behaviors were coded during 10-s intervals every 3 min during five 1-h periods (two light, three dark cycle) on postpartum days 2, 4, 6, and 8 in 36 unrelated C57BL/6J mothers. The distribution of mean frequencies/h for $\mathrm{PL}$, still crouched nursing, hovering over pups, self-grooming, and no contact with pups were determined. Offspring (6-12 weeks of age) from the eight mothers who exhibited the highest mean frequencies of $P L$ and the seven mothers who exhibited the lowest $P L$ frequencies underwent the following tests over three consecutive weeks: (1) elevated plus-maze (EPM) and 1-h open field on three successive days, (2) 3-h open field with an acute stressor (IP saline injection) at the 1-h time point, and (3) acoustic startle and prepulse inhibition. Females reared by low PL mothers exhibited significantly more time in the closed arms of the EPM, less locomotion, center time, and rearing during the first test in the open field, greater reactivity to an acute stressor, and reduced prepulse inhibition, an index of sensorimotor gating. Male offspring from low PL dams had reduced reactivity to an acute stressor, but no other altered performance in the behavioral tests. $\mathrm{PL}$ frequencies of $\mathrm{C} 57 \mathrm{BL} / 6 \mathrm{~J}$ mothers appear to selectively alter behavior outcomes, primarily in female offspring.

Keywords: maternal behavior, C57BL/6J, pup-licking, nursing, anxiety, prepulse inhibition, sensorimotor gating, stress

\section{INTRODUCTION}

Decades of research and clinical experience in primates and humans have shown that the amount and quality of nurturing received early in life strongly influences social competence, coping abilities, and vulnerability to mental illness later in life (Van Ijzendoorn, 1995; George and Solomon, 1999; Suomi, 1999; Repetti et al., 2002; Sameroff and Rosenblum, 2006; Weich et al., 2009). Adult offspring of Long Evans mothers that exhibit high, in contrast to low, levels of maternal licking during the early postnatal period exhibit less stress-induced anxiety-like behavior and hypothalamic-pituitary-adrenal axis activation, greater prepulse inhibition of acoustic startle and higher frequencies of pup-licking (PL) of their pups (Liu et al., 1997; Caldji et al., 1998; Francis et al., 1999; Champagne et al., 2003; Menard et al., 2004; Zhang et al., 2005). In these studies, most outcomes in adult offspring, other than maternal behavior, were assessed in males (Liu et al., 1997; Caldji et al., 1998; Menard et al., 2004; Zhang et al., 2005) although similar novel open field results were obtained in females (Francis et al., 1999). The consequences of high vs. low maternal licking may be mediated by differences in factors that influence the expression of specific genes in the brain (Weaver et al., 2004; Champagne, 2008; Zhang and Meaney, 2010).

There have been relatively few investigations in mice of the effects of early nurturing on the development of stress responses and social behavior. In-bred mouse strains exhibit considerable differences in various components of maternal behavior (Ward, 1980; Carlier et al., 1982; Brown et al., 1999; Priebe et al., 2005; Shoji and Kato, 2006; Champagne et al., 2007). A few investigators have taken advantage of the higher frequencies of PL and other components of maternal behavior in C57BL/6J or CBA/Ca compared to $\mathrm{BALB} / \mathrm{cJ}$ mothers to conduct cross-fostering experiments to examine the effects of variations in maternal nurturing on offspring development (Francis et al., 2003; Priebe et al., 2005; Shoji and Kato, 2009). BALB/cJ mice reared by C57BL/6J mothers exhibited less anxiety-like behavior than BALB/cJ mice reared by same strain mothers while $\mathrm{C} 57 \mathrm{BL} / 6 \mathrm{~J}$ mice reared by $\mathrm{BALB} / \mathrm{cJ}$ mothers did not differ from C57BL/6J mice reared by same strain mothers (Francis et al., 2003; Priebe et al., 2005). However, C57BL/6J mice 
reared by $\mathrm{BALB} / \mathrm{cJ}$ mothers were more anxious in the novel open field in one study (Priebe et al., 2005) but not in another (Francis et al., 2003). BALB/c mothers reared by CBA/Ca mothers exhibited more body licking of their own pups (but no differences in other components of maternal behavior) compared to BALB/c mothers reared by same strain dams (Shoji and Kato, 2009). Coutellier et al. (2008) found that lower crouched nursing and PL frequencies in C57BL/6 mothers that were required to forage for food were related to diminished anxiety-like behavior in male but not female offspring.

Only one other study has measured the range of frequencies of the components of maternal behavior within individual mouse strains (Champagne et al., 2007). To date, there are no published studies of within-strain variations in maternal behavior on the development of offspring. There are two compelling reasons to conduct studies in this area. First, behavior phenotyping of diverse mouse strains is a widely employed strategy for identifying animal models of psychiatric and neurodevelopmental disorders. Within-strain variations in maternal behavior could influence the results of tests used in behavior phenotyping projects. Second, investigation of variations in maternal behavior and their relationship to offspring outcomes within transgenic mouse strains may be an effective means of identifying genes involved in conveying the powerful epigenetic effects of early nurturing.

The goals of the current study were to (1) determine variability in the frequency of maternal behavior components in C57BL/6J dams and (2) compare behavioral outcomes in adult offspring of dams that exhibit high vs. low frequencies of PL.

\section{MATERIALS AND METHODS}

All experiments were conducted in accordance with the NIH Guide for the Care and Use of Laboratory Animals and were approved by the University of North Carolina Institutional Animal Care and Use Committee. All efforts were made to minimize the numbers of animals used and their suffering.

\section{ANIMALS}

Subjects were adult C57BL/6J mice and their offspring. Fifty 60day-old, nulliparous females and 10 males were obtained from Charles River Breeders. To maximize genetic and rearing variability, the females that were bred were all reared in different litters. Females were group housed 3-4 animals per cage and males were individually housed in standard Plexiglas cages $(19 \mathrm{~cm} \mathrm{~W}, 35 \mathrm{~cm}$ $\mathrm{L}, 14 \mathrm{~cm} \mathrm{H}$ ) with ad libitum access to water bottles and mouse chow pellets placed in recesses of the wire mesh lid of each cage. The ambient temperature was maintained at $24^{\circ} \mathrm{C}$ with lights on at $0700 \mathrm{~h}$ and lights off at $1900 \mathrm{~h}$.

For breeding, females that had been housed in the same cage were placed in the home cage of a male for 1 week. Copulatory efforts by males were confirmed by visual inspection during the dark phase. After the breeding period, females were removed from the male's cage and housed together in a clean cage. Beginning 14 days after females were first co-housed with males, they were checked daily to determine whether they were pregnant. When a female was confirmed to be pregnant, she was moved to her own home cage. Thirty-six females delivered and reared to weaning litters composed of 4-11 pups. This does not include females that failed to rear four or more healthy pups to weaning or lost more than two pups that were born alive.

\section{MATERNAL BEHAVIOR MEASUREMENTS}

Events in home cages were videotaped for $1.5 \mathrm{~h}$ during three light and two dark periods $(0530-0630,0715-0815,1200-1300,1615-$ 1715 , and $2345-0045 \mathrm{~h}$ ) beginning at $1200 \mathrm{~h}$ on postpartum days 2 (the day after pups were born), 4, 6, and 8, for a total of 20 recordings across the early neonatal period. Fluorescent red light bulbs provided illumination during the dark phase. Test cages were not disturbed over the 8-day postpartum period except for removal of dead newborns shortly after parturition and replenishing food and water on postpartum days 3 and 5 . Events in each cage were recorded through the front wall of the cage using a Panasonic BP330 black and white camera connected to a Panasonic PV-V402 VCR set on SLP mode that was programmed to record during the five daily time periods described above. Events occurring in each cage over each 24-h period were recorded on an 8-h videocassette (7.5 h total recording time). Cameras were mounted on horizontal arms clamped to a vertical pole of a shelf structure on which the VCRs were stacked. Each camera was placed so that the cage from which it was recording filled the entire field of view of the camera. A mirror was placed against the outer back side of each cage opposite to the camera so the reflection was recorded along with events in the cage. The mirror was tilted so that the reflection was from a somewhat elevated angle allowing a clear view of the mother's activities even when she was turned away from the camera and adjacent to the back wall of the cage. Because of technical errors, recordings were not obtained at all time periods for nine of the mothers: The number of missing recordings for those mothers are as follows: 5 from two mothers (all postpartum day 4 recordings from one mother and all postpartum day 6 recordings from another), 4 from one, 3 from one, 2 from three, and 1 from two.

Over a 1-h period beginning $15 \mathrm{~min}$ from the start of each $1.5 \mathrm{~h}$ of videotape, maternal and other behaviors were coded during 10 -s intervals every $3 \mathrm{~min}$ for a total of 20 observations/h and a maximum total of 400 observations over 20 behavior observation periods during the 4 postpartum days when behavior was videotaped. Behaviors coded during each interval were:

\section{Pup-licking}

At least one bout during the 10-s interval in which the mother licked a pup or pups two times or more in rapid succession.

\section{Self-grooming}

At least one bout during the 10-s interval in which the mother licked or chewed her fur or tail or rubbed her face/upper body with her forepaws. Hind leg scratching did not count.

\section{Still crouch}

The mother maintained a fixed upright nursing posture over pups with her ventrum elevated sufficiently so pups had easy access to her milk line. All four limbs remained in a fixed position and the mother did not engage in other behaviors during the entire 10 -s interval. 


\begin{abstract}
Hover
The mother remained in an upright nursing stance over pups throughout the 10-s interval with hind legs in a fixed position and her ventrum elevated sufficiently for pups to have access to her milk line but she engaged for some portion of the 10-s interval in other behaviors such as PL, self-grooming (SG), eating/drinking, etc.
\end{abstract}

\section{Arched-back nursing}

Frequency was the sum of still crouch (SC) and hover (HOV) frequencies.

\section{No pup contact}

Physical contact between the mother and one or more pups did not occur during the entire 10 -s interval.

\section{BEHAVIORAL MEASUREMENTS IN ADULT OFFSPRING Subject selection}

Litters reared by mothers that were in the top eight and bottom seven in the distribution of PL frequencies were studied between 6 and 12 weeks of age. A total of 64 offspring were tested (16 males and 16 females reared by low PL mothers, 15 males and 17 females reared by high-licking mothers). No more than three male or three female pups were tested from any one litter. Measures of adult offspring behavior were collected by observers who were blind to the rearing condition of the experimental subjects.

\section{Order of tests}

Mice were tested across 3 weeks. In the first week, mice were tested in the elevated plus-maze (EPM), and then given 1-h open field tests on three successive days. In the second week, mice were tested for reactivity to a brief stressor (an IP saline injection) during a single 3-h open field test. During the third week, mice were evaluated in the acoustic startle test.

\section{Elevated plus-maze}

Mice were given one 5-min trial on the plus-maze, which had two closed arms, with walls $40 \mathrm{~cm}$ in height, and two open arms (21 cm long). The maze was elevated $50 \mathrm{~cm}$ from the floor. Animals were placed on the center section $(9.5 \mathrm{~cm} \times 9.5 \mathrm{~cm})$, and allowed to freely explore the maze. Arm entries were defined as all four paws entering an arm. Entries and time in each arm were recorded during the trial by a human observer via computer coding.

\section{Open field activity}

Exploration in a novel environment was evaluated in photocellequipped automated open fields $(40 \mathrm{~cm} \times 40 \mathrm{~cm} \times 30 \mathrm{~cm}$; Versamax system, Accuscan Instruments). Measures were taken of total distance traveled, number of rearing movements, and time spent in the center during the test. Activity chambers were contained inside sound-attenuating boxes, equipped with houselights and fans. Mice were first given three 1 -h tests across 3 days. During the following week, mice were given a single 3 -h session. After the first $60 \mathrm{~min}$, the mouse was taken from the open field and given an IP injection, as a brief exposure to an aversive stimulus, and then returned to the chamber for another $2 \mathrm{~h}$.

\section{Acoustic startle test}

This procedure was conducted with a San Diego Instruments SRLab system, using published methods (Paylor and Crawley, 1997). Each test session consisted of 42 trials, presented following a 5-min habituation period. There were seven different types of trials: the no-stimulus trials, trials with the acoustic startle stimulus ( $40 \mathrm{~ms}$; $120 \mathrm{~dB}$ ) alone, and trials in which a prepulse stimulus $(20 \mathrm{~ms} ; 74$, $78,82,86$, or $90 \mathrm{~dB}$ ) preceded the startle stimulus by $100 \mathrm{~ms}$. The different trial types were presented in blocks of seven, in randomized order within each block, with an average inter-trial interval of 15-s. Measures were taken of the startle amplitude for each trial, defined as the peak response during a $65-\mathrm{ms}$ sampling window that began with the onset of the startle stimulus. Levels of prepulse inhibition at each prepulse sound level were calculated as: 100 -[(response amplitude for prepulse stimulus and startle stimulus/response amplitude for startle stimulus alone) $\times 100$ ].

\section{STATISTICAL ANALYSIS}

Data were analyzed using two-way or repeated measures analysis of variance (ANOVA) examining effects of maternal licking frequency (low and high) and sex of offspring. Repeated measures included type of arm (open or closed; EPM), day of testing and 5-min interval (open field), 10-min period pre- and post-injection (reactivity to acute stressor), and stimulus decibel level (acoustic startle test). Because the factor of sex was significant for almost every test, separate ANOVAs were then run for male and female animals. Data were further analyzed using litter size (number of pups) as a covariate. Fisher's protected least-significant difference (PLSD) tests were used for comparing group means when a significant $F$ value for maternal licking (main effect or interaction) was determined. For all comparisons, significance was set at $p<0.05$.

\section{RESULTS}

The distributions of all mothers' PL, SC, and HOV frequencies are illustrated in Figures 1-3. The frequencies are presented as mean scores per $\mathrm{h}$ of observation (maximum possible score $=20$ ) rather than mean scores per day or over the 4-days of observations because behavior was not recorded during some observation periods for nine mothers. The range of PL scores was from 2.60 to 5.88 (frequencies of 13-29.4\%) and was skewed toward the lower end of the range. SC and HOV scores exhibited a more normal distribution and ranged, respectively, from 7.2 to 11.0 (frequencies of $36-55 \%$ ) and from 3.7 to 6.5 (frequencies of $18.5-32.5 \%$ ).

Means \pm SEM of maternal and other behavior frequencies in the mothers whose PL frequencies (PL/litter) were in the top eight and bottom seven are summarized in Table 1.

\section{ELEVATED PLUS-MAZE}

The plus-maze test was used as a standard measure of anxietylike behavior in rodents. A significant main effect of maternal licking was found for time spent on the open and closed arms during the 5 -min test $[F(1,60)=16.22, p=0.0002]$. As shown in Figure 4, the female mice from the low-licking dams spent more time in the relative safety of the closed arms, in comparison to the offspring of high-licking dams. There were no differences in the male groups for arm time. In addition, there were no significant effects of maternal licking on any of the other measures recorded 


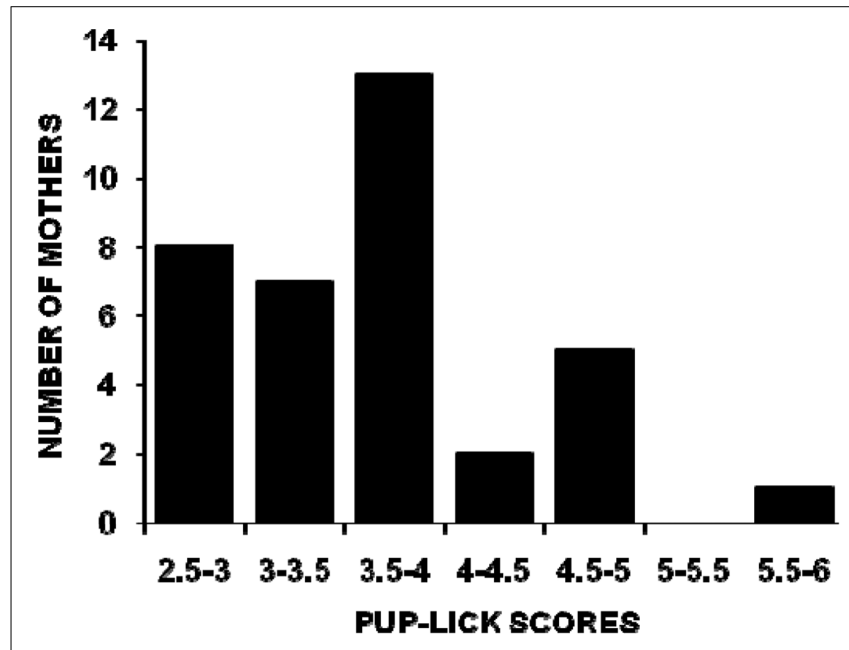

FIGURE 1 | The distribution of mean hourly pup-licking scores (maximum possible score/ $\mathrm{h}=20$ ) for $36 \mathrm{C57BL} / 6 \mathrm{~J}$ mothers over five 1-h observations (two light phase, three dark phase) on postpartum days $2,4,6$, and 8 .

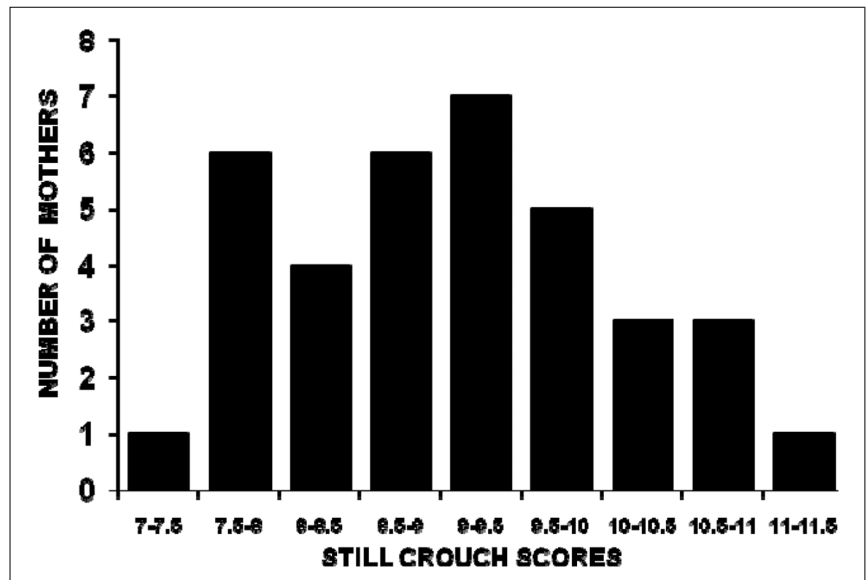

FIGURE 2 |The distribution of mean hourly still crouch nursing scores (maximum possible score/ $\mathrm{h}=20$ ) for $36 \mathrm{C57BL} / 6 \mathrm{~J}$ mothers over five 1-h observations (two light phase, three dark phase) on postpartum days $2,4,6$, and 8 .

for the test, including percent time and percent entries into the open arms.

\section{ACTIVITY IN AN OPEN FIELD}

Mice were given three $1-\mathrm{h}$ tests across 3 days, in order to observe patterns of exploration and habituation within and across sessions. The results indicated that the amount of PL received had significant effects in the open field test, but only in female offspring. An overall ANOVA revealed a complex 3-way interaction between maternal licking, sex of offspring, and day of testing for distance traveled $[F(2,120)=5.78, p=0.004]$. Separate analyses for each sex indicated that maternal licking had a significant effect on distance traveled by the female offspring (Figure 5). In the female mice, the offspring from the low-licking dams had significantly less exploration during the initial introduction to the novel environment, but had higher rates of locomotion by the third

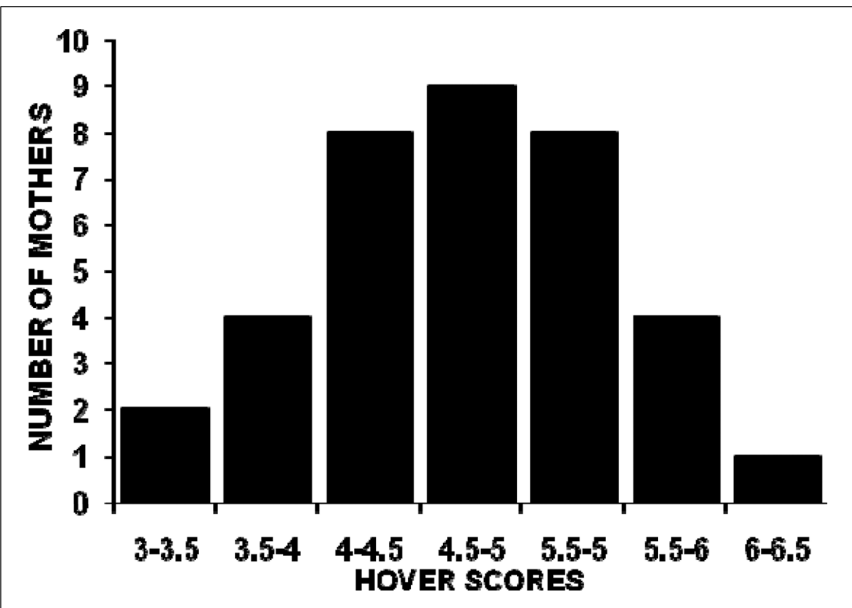

FIGURE 3 |The distribution of mean hourly hover over pups scores (maximum possible score $/ \mathrm{h}=20$ ) for $36 \mathrm{C57BL} / 6 \mathrm{~J}$ mothers over five 1-h observations (two light phase, three dark phase) on postpartum days $2,4,6$, and 8 .

day of testing [post hoc analyses following maternal licking $\times$ day interaction, $F(2,62)=6.51, p=0.0027$; and maternal licking $\times 5$ min interval interaction, $F(11,341)=1.83, p=0.0485]$. The group differences on Day 3 suggested that the female mice in the lowlick group did not show the typical pattern of habituation across days. In line with this observation, the female mice in the highlicking group had significant decreases in activity across the days of testing [within-group repeated measures ANOVA, main effect of day, $F(2,32)=10.51, p=0.0003$; day $\times$ interval interaction, $F(22,352)=2.9, p<0.0001$ ], while there were no significant effects of day in the female mice from the low-licking group.

Similar to the measure for locomotion, an overall ANOVA revealed a three-way interaction between maternal licking, sex of offspring, and day of testing for rearing movements $[F(2,120)=3.23, p=0.0429]$. Separate analyses for each sex indicated an overt difference between the female groups for vertical activity (Figure 6). Rearing movements in the low-lick female offspring were markedly decreased across most of the first test, although this difference was only observed on Day 1 [maternal licking $\times$ day interaction, $F(2,62)=7.76, p=0.001]$. The reduced rate of rearing could indicate higher anxiety-like behavior in the low-lick group (Choleris et al., 2001; Takahashi et al., 2008).

Time in the center region of the open field was used as an index of anxiety-like behavior (Figure 7). An overall ANOVA indicated a three-way interaction between maternal licking, sex of offspring, and session interval $[F(11,660)=2.34, p=0.0079]$. On Day 1, the female offspring of the low-licking dams spent significantly less time in the center region, suggesting that this group had higher anxiety in the open field test [maternal licking $\times$ interval interaction, $F(11,341)=3.29, p=0.0003]$. As with the other activity measures, no significant differences were observed in the male groups.

\section{REACTIVITY TO AN ACUTE STRESSOR}

In the week following the first three activity tests, mice were evaluated for response to a brief aversive stimulus (an IP injection of saline), given after the first h of a 3-h test (Figure 8). Because 
Table 1 | Low vs. high pup-licking mothers: means ( \pm SEM) of pups/litter and maternal behaviors.

\begin{tabular}{|c|c|c|c|c|c|c|c|}
\hline Mothers & Pups/litter & PL & SG & SC & HOV & ABN & NPC \\
\hline Low PL & $7.9 \pm 0.7$ & $2.8 \pm 0.1^{*}$ & $2.1 \pm 0.2$ & $9.5 \pm 0.4$ & $3.9 \pm 0.2^{*}$ & $13.4 \pm 0.3$ & $3.5 \pm 0.4$ \\
\hline High PL & $6.9 \pm 0.7$ & $4.8 \pm 0.2$ & $1.9 \pm 0.2$ & $8.6 \pm 0.2$ & $5.4 \pm 0.2$ & $14.0 \pm 0.2$ & $3.4 \pm 0.4$ \\
\hline
\end{tabular}

${ }^{*} p<0.001$, Low vs. High PL. PL, pup-lick; SG, self-groom; SC, still crouch; HOV, hover; ABN, arched-back nursing= sum of SC and HOV; NPC, no pup contact.
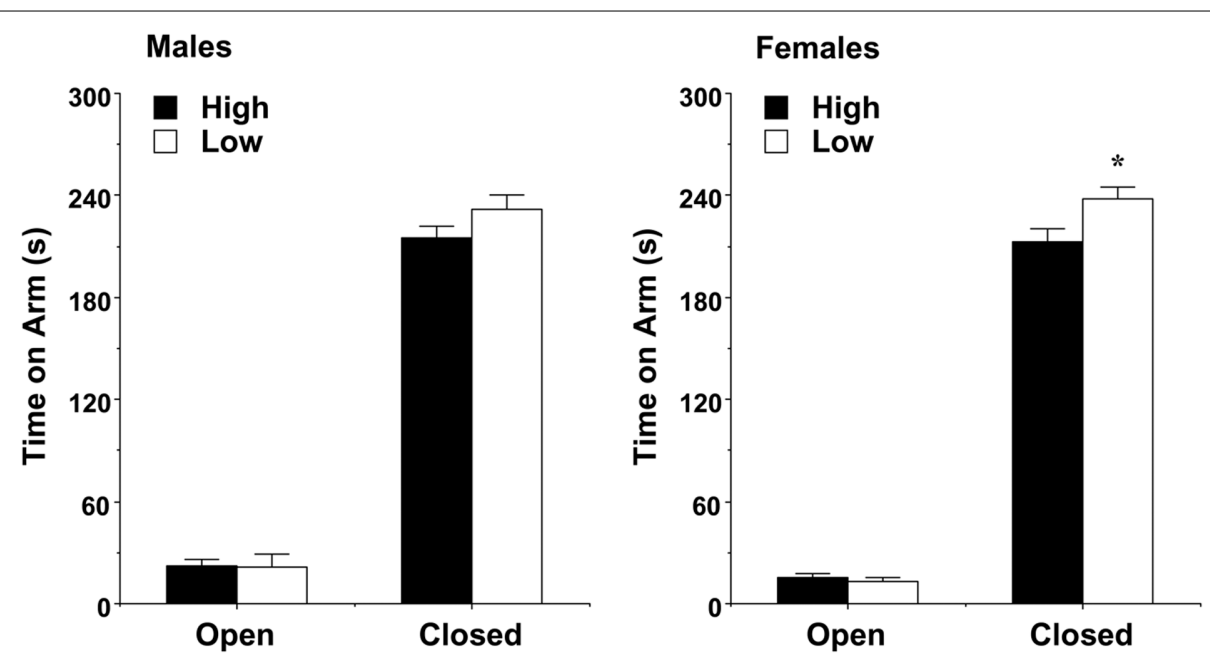

FIGURE 4 |Time spent on the open and closed arms of the elevated plus-maze (mean + SEM) during a 5-min test. Female offspring of dams with low levels of pup-licking spent significantly more time in the closed arms than the offspring of high-licking dams. ${ }^{*} p<0.05$.

general exploration in the open field had already been investigated in the previous three tests, the analysis focused on changes in activation following the stressor. A repeated measures ANOVA on distance traveled during the 10-min pre-injection and postinjection time points revealed a three-way interaction between maternal licking, sex, and time $[F(1,59)=4.78, p=0.0328]$. Separate analyses indicated different effects of maternal licking in the male and female groups. In the male mice, there were no differences in locomotion before the injection. However, after the brief stressor, the mice from the low-licking dams had minimal activation, in comparison to the offspring from high-licking dams [maternal licking $\times$ time interaction, $F(1,29)=7.58, p=0.0101$ ] In the female groups, increased rates of locomotion were observed in the low-lick offspring both before and after the stressor [main effect of licking, $F(1,30)=8.81, p=0.0058$ ].

With the measure for rearing movements, the pattern of activation following the stressor was opposite in the male and female groups, with the male mice from low-lick dams showing less activation [maternal licking $\times$ time interaction, $F(1,29)=5.97, p=0.0208]$, and the female mice from low-lick dams having greater activation [maternal licking $\times$ time interaction, $F(1,30)=7.82, p=0.0089]$.

\section{ACOUSTIC STARTLE TEST}

Levels of maternal licking did not have any significant effects on amplitude of startle responses, indicating that the groups had comparable reactivity to the acoustic stimuli. However, differences between the female groups were found for prepulse inhibition, an index of sensorimotor gating (Figure 9). An overall repeated measures ANOVA revealed a significant main effect of maternal licking $[F(1,60)=4.73, p=0.0337]$ and maternal licking $\times$ sex interaction $[F(1,60)=4.67, p=0.0346]$. Separate analyses for each sex showed that the female mice from the low-licking group had reduced percent inhibition at every prepulse sound level [post hoc analyses following significant main effect of maternal licking, $F(1,31)=10.33, p=0.003]$. In contrast, no differences in prepulse inhibition were found in the male groups.

\section{ANALYSIS WITH LITTER SIZE AS COVARIATE}

The use of number of pups per litter as a covariate did not reveal additional significant effects of maternal licking on offspring behavior. It is possible that this approach was not more informative because of the low variability in pup number per litter. This was partially due to the elimination of any litters with less than four pups. Overall, the mean number of pups in the low-lick litters did not differ significantly from the mean number in the high-lick group [Table $1 ; F(1,13)=1.02, p=0.3301$ ].

\section{DISCUSSION}

This is the first study to compare behavioral outcomes between adult offspring of mothers of a single mouse strain (C57BL/6J) that exhibited frequencies of PL at the high vs. low end of the distribution. Compared to adult females that received high frequency maternal licking in infancy, adult females that received low frequency maternal licking exhibited significantly (1) more 

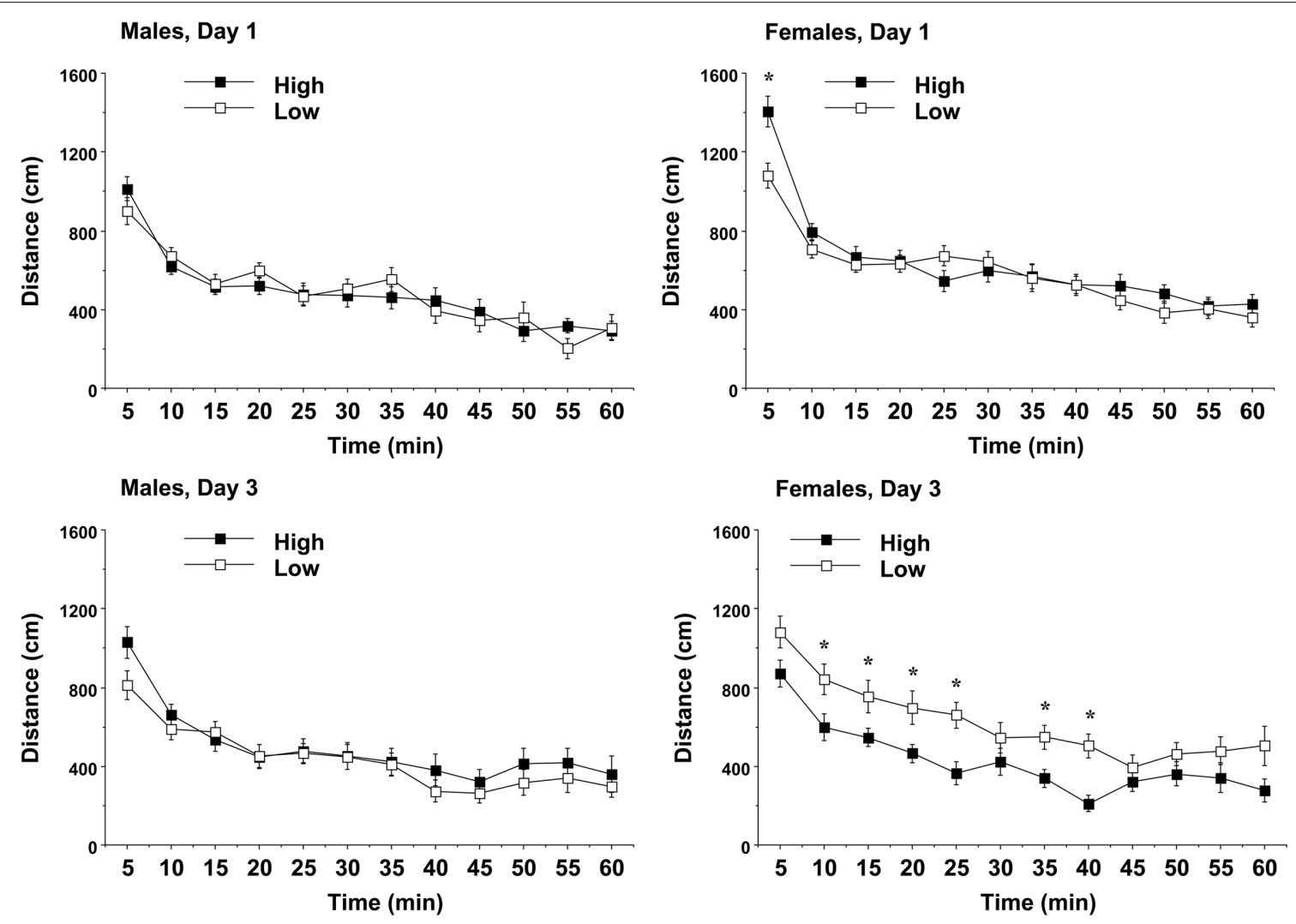

FIGURE 5 | Locomotion in a novel environment during a 1-h session. Data shown are means ( \pm SEM) for the first (Day 1) and third (Day 3 ) tests. Female offspring of dams with low levels of pup-licking had significantly lower locomotion on Day 1, but higher locomotion on Day 3 , than the offspring of high-licking dams. ${ }^{*} p<0.05$

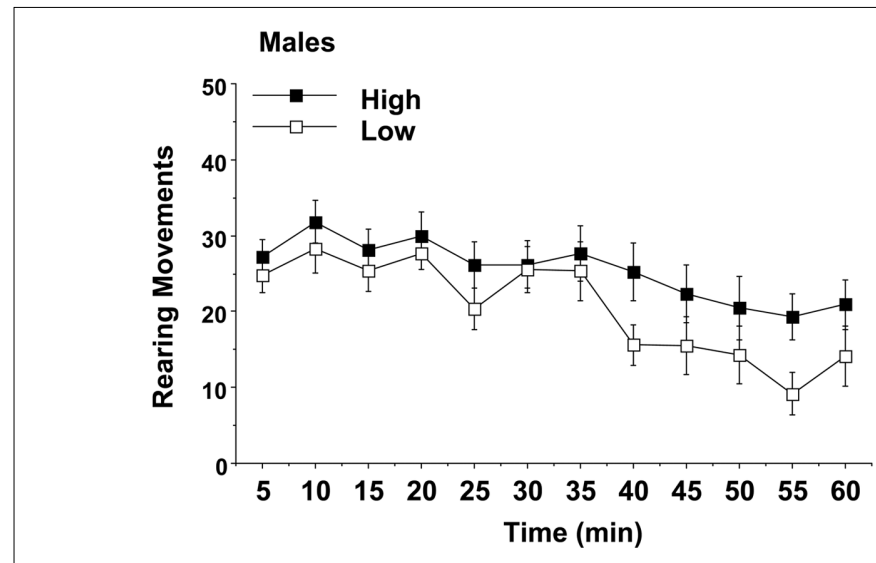

FIGURE 6 | Vertical activity in a novel environment during a 1-h session. Data shown are means ( \pm SEM) for the first (Day 1 ) test. Female offspring of

anxiety-like behavior in the novel open field test, (2) no reduction in locomotion over repeated testing in the open field, suggesting impaired habituation to the test situation, (3) increased reactivity after an acute stressor (a saline injection), and (4) deficits in prepulse inhibition. There were no significant behavioral differences between adult male C57BL/6J mice reared by high vs. low PL

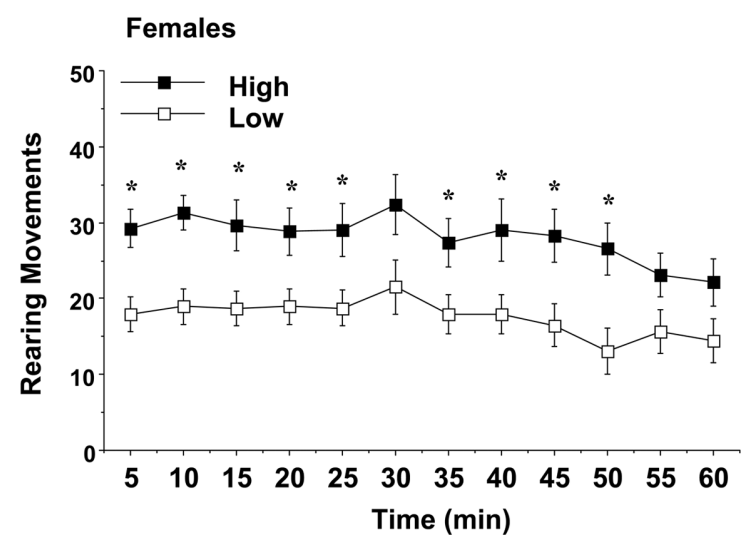

dams with low levels of pup-licking had significantly lower rearing movements than the offspring of high-licking dams. ${ }^{*} p<0.05$.

mothers on most tests, although the male offspring of low-licking dams had less reactivity to the brief stressor.

Comparisons of our results with the limited number of previous studies in mice examining the effects of maternal behavior variation on adult outcomes in C57BL/6J offspring are hampered by considerable methodological differences among 


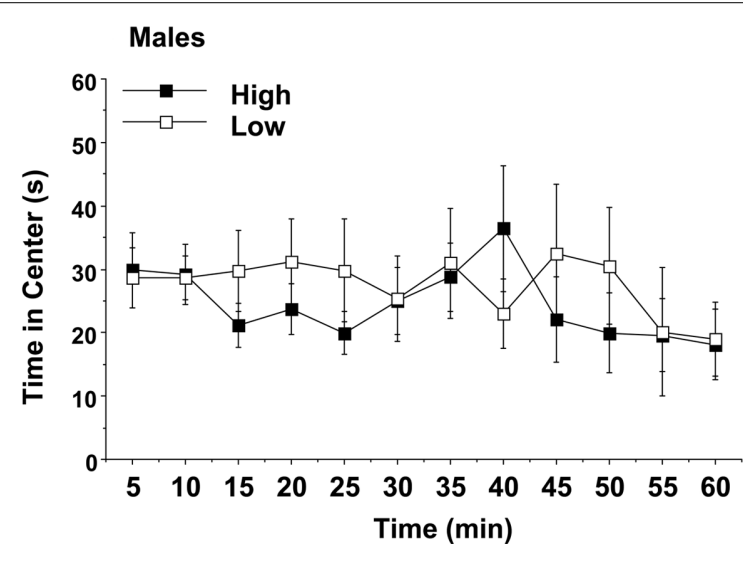

FIGURE 7 |Time spent in the center region of a novel environment during a 1-h session. Data shown are means $( \pm S E M)$ for the first (Day 1$)$ test.

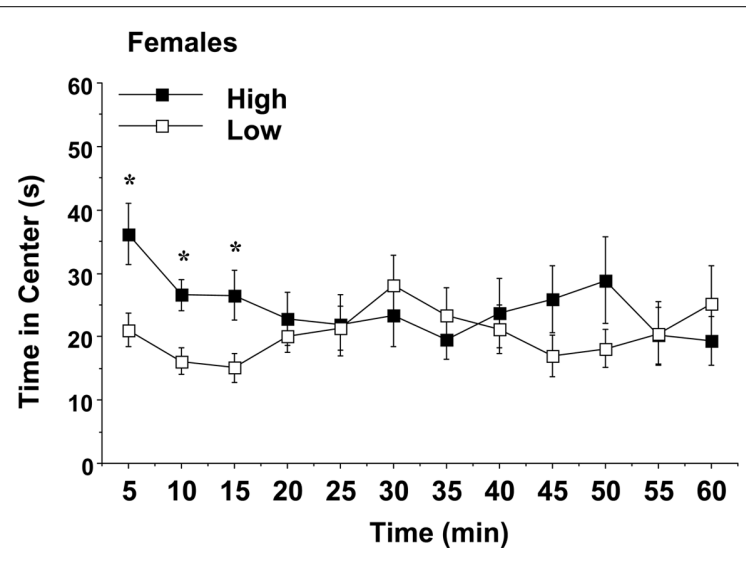

Female offspring of dams with low levels of pup-licking spent significantly less time in the center than the offspring of high-licking dams. ${ }^{*} p<0.05$. research groups. Francis et al. (2003) cross-gestated embryos or cross-fostered newborns between C57BL/6J and BALB/cJ mothers. Priebe et al. (2005) also cross-fostered neonates between these strains and confirmed previous reports that C57BL/6J mothers exhibit significantly more PL than BALB/cJ mothers. Outcomes were assessed in adult male but not female offspring. Both groups found no differences between C57BL/6J mice reared by BALB/cJ mothers and $\mathrm{C} 57 \mathrm{BL} / 6 \mathrm{~J}$ reared by same strain mothers in the EPM and prepulse inhibition tests. There were no differences in novel open field behavior as well in one study (Francis et al., 2003) but C57BL/6J mice reared by BALB/cJ mothers exhibited significantly less center time in that test (Priebe et al., 2005). With the exception of the latter finding, these results are in agreement with our data indicating there are no effects of PL variations on behavior outcomes in male $\mathrm{C} 57 \mathrm{BL} / 6 \mathrm{~J}$ mice. It is unfortunate that female offspring were not evaluated in these studies. Coutellier et al. (2008) were able to decrease PL frequencies (on days 1 and 2 but not subsequent postpartum days) and crouched nursing frequencies (on postpartum days 1-4) in C57BL/6J mothers by requiring them to forage for food. Adult offspring of foraging compared to non-foraging mothers exhibited no differences in open field behavior, but increased locomotion and a trend toward more open arm time in the elevated zero maze in males, but not females. These results contrast with the significant relationships we found in adult female, but not male, $\mathrm{C} 57 \mathrm{BL} / 6 \mathrm{~J}$ offspring between frequencies of PL received and measures of anxiety. The effects on behavioral development of foraging-induced and natural variations in maternal behavior may be substantial.

In rats, adult offspring that received high compared to low frequencies of maternal licking during the early postnatal period exhibited less anxiety-like behavior in the open field and other tests, as well as greater prepulse inhibition (Caldji et al., 1998; Francis et al., 1999; Zhang et al., 2005). These results, which were mostly obtained from male offspring, contrast with our findings that variations in PL are mainly related to behavior in adult female, but not male, C57BL/6J offspring. However, the one rat study that examined female offspring obtained results similar to ours; recipients of high compared to low maternal licking spent significantly more time in the center of the novel open field (Francis et al., 1999). Two earlier studies (Gubernick and Alberts, 1985; Moore et al., 1997) reported that male rat pups received significantly more maternal licking than female pups. More recently, Champagne et al. (2003) found no significant difference between the frequencies at which male and female pups were licked although the mean frequency for males was slightly higher. Greater frequency or less variability in the frequency of PL directed toward C57BL/6J male pups may explain why we found no difference in behavior outcomes in adult male offspring reared by high vs. low PL mothers.

Only one other study has examined the range of frequencies of components of maternal behavior in C57BL/J6 mice (Champagne et al., 2007), although other groups studied the mean frequencies of behaviors in C57BL/6J mothers (Ward, 1980; Anisman et al., 1998; Brown et al., 1999; Priebe et al., 2005; Shoji and Kato, 2006; Carola and Gross, 2010). The range of PL frequency in our study (13-29.4\%) differs considerably from the range reported by Champagne et al. (2007); 3-14\%). The mean PL frequency in this study $(18.3 \%)$ fits in the upper range of the considerable variation in PL frequencies (approximately 4-22\%) measured in other studies of C57BL/6J mothers cited above. It is difficult to attribute this strikingly wide range of PL frequencies solely to methodological differences among studies. PL frequencies may naturally vary substantially among C57BL/6J mothers reared and bred in different animal colonies.

This study has several limitations. Offspring were tested over a wide age range (6-12 weeks). The numbers of male and female pups were not recorded for three of the litters reared by high PL mothers. Some of the litters had very uneven sex distributions: e.g., one low PL litter had five male and no female pups and one high PL litter had four female pups and one male. Incomplete data on sex distribution in test litters prevents us from examining the influence of this factor on behavior outcomes. Furthermore, the estrus state of females was not assessed so this could not be entered into the analysis as a covariate. Because of procedural errors and equipment failure the complete 

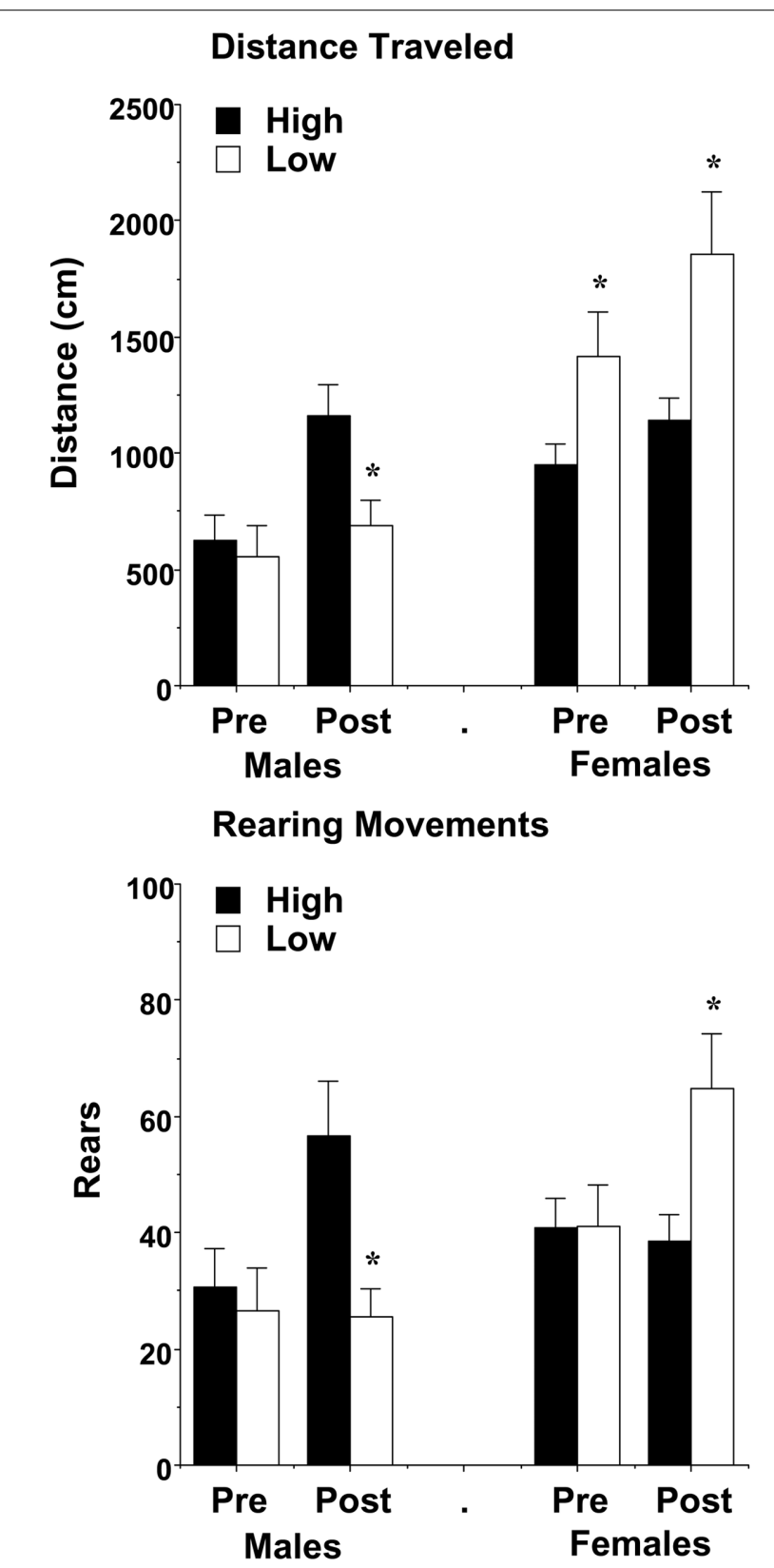

FIGURE 8 | Locomotion and vertical activity before and following exposure to an aversive stimulus (a saline injection). Data shown are means $( \pm$ SEM) for the 10-min period pre- and post-injection. In comparison to offspring of dams with high levels of pup-licking, female offspring of low-licking dams had increased activity, while the male offspring had decreased activity, during the test. ${ }^{*} p<0.05$.

set of video recordings of maternal behavior were not obtained in 9 of 36 dams. Determination of which dams exhibited high and low end PL frequencies may have been affected by these missing data.

Comparative behavior phenotyping of mouse strains is becoming a widely employed strategy to identify animal models of psychiatric and neurodevelopmental disorders (Moy et al., 2007; Kalueff et al., 2008). The behavior measures in which we found

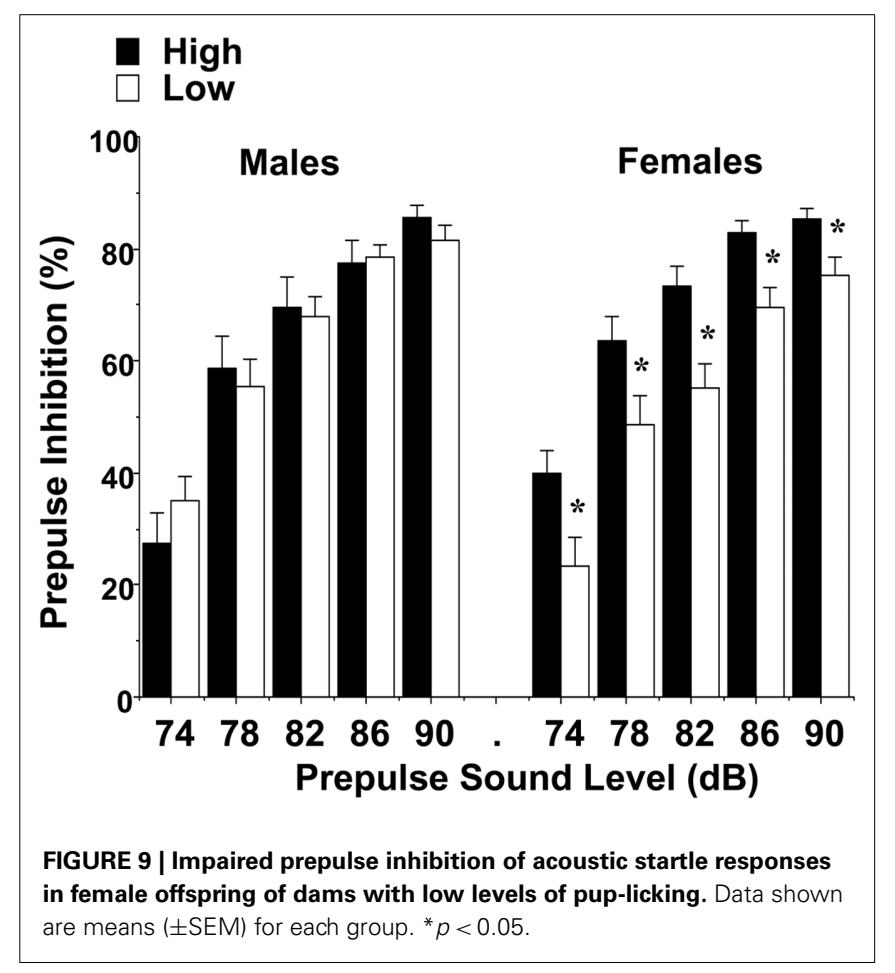

significant differences between mice that received high vs. low PL (EPM, open field, PPI) are commonly used in these studies. Published comparisons among separate research centers have reported considerable differences in behavior test results within-strains, even with strenuous efforts to standardize sources of animals, animal handling, test procedures and test apparati (Crabbe et al., 1999; Wahlsten et al., 2003; Mandillo et al., 2008). Data from open field, EPM, and PPI tests were far more variable withinstrains among research centers than data from other tests (e.g., water maze, alcohol preference). Our results raise concerns that differences among separate laboratories in maternal behavior levels within mouse strains may influence behavior phenotyping outcomes. However, our findings that PL variations are primarily related to behavior outcomes in female offspring bring into question the influence of variations in maternal behavior on the outcomes of mouse behavior phenotyping studies. In studies comparing research centers, differences in behavior test results were significant for both sexes (Crabbe et al., 1999; Wahlsten et al., 2003). Nonetheless, our results suggest that measures of maternal behavior received during the postnatal period may be important covariates that may improve assessment of the validity of mouse models of human behavioral and emotional disorders. Conducting such studies in transgenic mice may also identify genes that are involved in the epigenetic effects of maternal behavior on specific adult behavior outcomes. Unfortunately, quantification of maternal behavior is very laborious and not conducive to the high throughput scale of most mouse behavior phenotyping projects.

\section{ACKNOWLEDGMENTS}

This project was supported by NIMH grant R01 MH061995 (Cort A. Pedersen) and NICHD grant P30 HD03110 (Dr. Joe Piven). 


\section{REFERENCES}

Anisman, H., Zaharia, M. D., Meaney, M. J., and Meralis, Z. (1998). Do early-life events permanently alter behavioral and hormonal responses to stressors? Int. J. Dev. Neurosci. 16, 149-164.

Brown, R. E., Mathieson, W. B., Stapleton, J., and Neumann, P. E. (1999). Maternal behavior in female C57BL/6J and DBA/2J inbred mice. Physiol. Behav. 67, 599-605.

Caldji, C., Tannenbaum, B., Sharma, S., Francis, D., Plotsky, P. M., and Meaney, M. J. (1998). Maternal care during infancy regulates the development of neural systems mediating the expression of fearfulness in the rat. Proc. Natl. Acad. Sci. U.S.A. 95, 5335-5340.

Carlier, M., Roubertoux, P., and CohenSalmon, C. (1982). Differences in patterns of pup care in Mus musculus domesticus. 1. Comparisons between eleven inbred strains. Behav. Neural Biol. 35, 205-210.

Carola, V., and Gross, C. (2010). BDNF moderates early environmental risk factors for anxiety in mouse. Genes Brain Behav. 9, 379-389.

Champagne, F. A. (2008). Epigenetic mechanisms and the transgenerational effects of maternal care. Front. Neuroendocrinol. 29:3. doi: 10.1016/j.yfrne.2008.03.003

Champagne, F. A., Curley, J. P., Keverne, E. B., and Bateson, P. P. G. (2007). Natural variations in postpartum maternal care in inbred and outbred mice. Physiol. Behav. 91, 325-334.

Champagne, F. A., Francis, D. D., Mar, A., and Meaney, M. J. (2003). Naturally-occurring variations in maternal care in the rat as a mediating influence for the effects of environment on the development of individual differences in stress reactivity. Physiol. Behav. 79, 359-371.

Choleris, E., Thomas, A. W., Kavaliers, M., and Prato, F. S. (2001). A detailed ethological analysis of the mouse open field test: effects of diazepam, chlordiazepoxide and an extremely low frequency pulsed magnetic field. Neurosci. Biobehav. Rev. 25, 235-260.

Coutellier, L., Friedrich, A.-C., Failing, K., and Würbel, H. (2008). Variations in the postnatal maternal environment in mice: effects on maternal behavior and behavioural and endocrine responses in the adult offspring. Physiol. Behav. 93, 395-407.

Crabbe, J. C., Wahlsten, D., and Dudek, B. C. (1999). Genetics of mouse behavior: interactions with laboratory environment. Science 284, 1670-1672.

Francis, D. D., Diorio, J., Liu, D., and Meaney, M. J. (1999). Nongenomic transmission across generations of maternal behavior and stress responses in the rat. Science 286, 1155-1158.

Francis, D. D., Szegda, K., Campbell, G., Martin, W. D., and Insel, T. R. (2003), Epigenetic sources of behavioral differences in mice. Nat. Neurosci. 6, 445-446.

George, C., and Solomon, J. (1999). "Attachment and caregiving: the caregiving behavioral system," in Handbook of Attachment: Theory, Research, and Clinical Applications, eds J. Cassidy and P. R Shaver (New York: The Guilford Press), 649-670.

Gubernick, D. J., and Alberts, J. R. (1985). Maternal licking by virgin and lactating rats: water transfer from pups. Physiol. Behav. 34, 501-506.

Kalueff, A. V., Ren-Patterson, R. F., LaPorte, J. L., and Murphy, D. L. (2008). Domain interplay concept in animal models of neuropsychiatric disorders: a new strategy for high-throughput neurophenotyping research. Behav. Brain Res. 188, 243-249.

Liu, D., Diorio, J., Tannenbaum, B., Caldji, C., Francis, D., Freedman, A., Sharma, S., Pearson, D., Plotsky, P. M., and Meaney, M. J. (1997). Maternal care, hippocampal glucocorticoid receptors, and hypothalamicpituitary-adrenal responses to stress. Science 277, 1659-1662.

Mandillo, S., Tucci, V., Hölter, S. M., Meziane, H., Banchaabouchi, M. A., Kallnik, M., Lad, H. V., Nolan, P. M., Ouagazzal, A. M., Coghill, E. L., Gale, K., Golini, E., Jacquot, S., Krezel, W., Parker, A., Riet, F., Schneider, I., Marazziti, D., Auwerx, J., Brown, S. D., Chambon, P., Rosenthal, N., Tocchini-Valentini, G., and Wurst, W. (2008). Reliability, robustness, and reproducibility in mouse behavioral phenotyping: a crosslaboratory study. Physiol. Genomics 34, 243-255.

Menard, M. J., Champagne, F., and Meaney, M. J. (2004). Maternal care alters behavioral and neural activity patterns in the defensive burying paradigm. Neuroscience 129, 297-308.

Moore, C. L., Wong, L., Daum, M. C., and Leclair, O. U. (1997). Motherinfant interactions in two strains of rats: implications for dissociating mechanism and function of a maternal pattern. Dev. Psychobiol. 30, 301-312.

Moy, S. S., Nadler, J. J., Young, N. B., Perez, A., Holloway, L. P., Barbaro, R. P., Barbaro, J. R., Wilson, L. M., Threadgill, D. W., Lauder, J. M., Magnuson, T. R., and Crawley, J. N. (2007). Mouse behavioral tasks relevant to autism: phenotypes of 10 inbred strains. Behav. Brain Res. 176, 4-20.

Paylor, R., and Crawley, J. N. (1997). Inbred strain differences in prepulse inhibition of the mouse startle response. Psychopharmacology (Berl.) 132, 169-180.

Priebe, K., Romeo, R. D., Francis, D. D., Sisti, H. M., Mueller, A., McEwen, B. S., and Brake, W. G. (2005). Maternal influences on adult stress and anxiety-like behavior in C57BL/6J and BALB/cJ mice: a cross-fostering study. Dev. Psychobiol. 47, 398-407.

Repetti, R. L., Taylor, S. E., and Seeman, T. E. (2002). Risky families: family social environments and the mental and physical health of offspring. Psychol. Bull. 128, 330-366.

Sameroff, A. J., and Rosenblum, K. L. (2006). Psychosocial constraints on the development of resilience. Ann. N. Y. Acad. Sci. 1094, 116-124.

Shoji, H., and Kato, K. (2006). Maternal behavior of primiparous females in inbred strains of mice: a detailed descriptive analysis. Physiol. Behav. 89, 320-328.

Shoji, H., and Kato, K. (2009). Maternal care affects the development of maternal behavior in inbred mice. Dev. Psychobiol. 51, 345-357.

Suomi, S. J. (1999). "Attachment in rhesus monkeys," in Handbook of Attachment: Theory, Research, and Clinical Applications, eds J. Cassidy and P. R Shaver (New York: The Guilford Press), 181-197.

Takahashi, A., Nishi, A., Ishii, A., Shiroishi, T., and Koide, T. (2008). Systematic analysis of emotionality in consomic mouse strains established from $\mathrm{C} 57 \mathrm{BL} / 6 \mathrm{~J}$ and wild-derived MSM/Ms. Genes Brain Behav. 7, 849-858.

Van Ijzendoorn, M. (1995). Adult attachment representations, parental responsiveness, and infant attachment: a meta-analysis on the predictive validity of the adult attachment interview. Psychol. Bull. 117, 387-403.

Wahlsten, D., Metten, P., Phillips, T. J., Boehm, S. L. II, Burkhart-Kasch, S., Dorow, J., Doerksen, S., Downing, C., Fogarty, J., Rodd-Henricks, K., Hen, R., McKinnon, C. S., Merrill, C. M., Nolte, C., Schalomon, M.,
Schlumbohm, J. P., Sibert, J. R., Wenger, C. D., Dudek, B. C., and Crabbe, J. C. (2003). Different data from different labs: lessons from studies of gene-environment interaction. J. Neurobiol. 54, 283-311.

Ward, R. (1980). Some effects of strain differences in the maternal behavior of inbred mice. Dev. Psychobiol. 13, 181-190.

Weaver, I. C. G., Cervoni, N., Champagne, F., D’Alessio, A. C., Sharma, S., Seckl, J. R., Dymov, S., Szyf, M., and Meaney, M. J. (2004). Epigenetic programming by maternal behavior. Nat. Neurosci. 7, 847-854.

Weich, S., Patterson, J., Shaw, R., and Stewart-Brown, S. (2009). Family relationships in childhood and common psychiatric disorders in later life: systemic review of prospective studies. Br. J. Psychiatry 194, 392-398.

Zhang, T. Y., Chrétien, P., Meaney, M. J., and Gratton, A. (2005). Influence of naturally occurring variations in maternal care on prepulse inhibition of acoustic startle and the medial prefrontal cortical dopamine response to stress in adult rats. $J$. Neurosci. 25, 1493-1502.

Zhang, T. Y., and Meaney, M. J. (2010). Epigenetics and the environmental regulation of the genome and its function. Annu. Rev. Psychol. 61, 439-466, C1-C3.

Conflict of Interest Statement: The authors declare that the research was conducted in the absence of any commercial or financial relationships that could be construed as a potential conflict of interest.

Received: 07 March 2011; paper pending published: 17 March 2011; accepted: 29 June 2011; published online: 11 July 2011. Citation: Pedersen CA, Vadlamudi S, Boccia ML and Moy SS (2011) Variations in maternal behavior in $C 57 B L / 6 J$ mice: behavioral comparisons between adult offspring of high and low puplicking mothers. Front. Psychiatry 2:42. doi: 10.3389/fpsyt.2011.00042

This article was submitted to Frontiers in Child and Neurodevelopmental Psychiatry, a specialty of Frontiers in Psychiatry. Copyright () 2011 Pedersen, Vadlamudi, Boccia and Moy. This is an open-access article subject to a non-exclusive license between the authors and Frontiers Media $S A$, which permits use, distribution and reproduction in other forums, provided the original authors and source are credited and other Frontiers conditions are complied with. 